\title{
The relationship between intelligence and creativity: On methodology for necessity and sufficiency
}

\author{
Michael John Ilagan and Welfredo Patungan
}

School of Statistics, University of the Philippines Diliman, Philippines

Version 2019/09/12. This paper is not the copy of record and may not exactly repli-
cate the authoritative document published in the APA journal. Please do not copy or
cite without author's permission. The final article is available, upon publication, at:
http://dx.doi.org/10.1037/arc0000050

\begin{abstract}
On the relationship between intelligence and creativity, a classic theory is that intelligence is necessary but not sufficient for creativity. Graphically, this theory is represented by a triangular shape of bivariate scatter between the two. As conventional linear methods are known to be inappropriate, a long-standing problem has been how to substantiate this theory. One innovation purported to solve this problem is the use of Necessary Condition Analysis (NCA), a method that confirms the relationship on the basis of an empty upper left corner in the scatterplot. The present article elaborates a novel take on this methodological problem. What it takes to account for necessity and sufficiency is tackled, and it is argued that NCA is not an appropriate method. As an alternative, a probability model of creativity as a function of IQ was posited, in particular for double-bounded creativity variables. Using the model proposed, intelligence vs. creativity data from Jauk et al. (2013b) were reanalyzed. A formal hypothesis based on the theorized relationship was supported for one of the two creativity variables analyzed.
\end{abstract}

Keywords: beta distribution, creativity, intelligence, quantile regression, Necessary Condition Analysis

The authors thank Emanuel Jauk, Mathias Benedek, Beate Dunst, and Aljoscha Neubauer for sharing their data and answering queries regarding their work.

The authors have made available for use by others the data (see Jauk, Benedek, Dunst, \& Neubauer, 2013a) that underlie the analyses presented in this paper, thus allowing replication and potential extensions of this work by qualified researchers. Next users are obligated to involve the data originators in their publication plans, if the originators so desire.

Correspondence concerning this article should be addressed to Michael Ilagan, who is now at the Department of Mathematics and Statistics, University of Calgary, Calgary, AB, Canada. Email: michaeljohn.ilagan@ucalgary.ca 


\section{Introduction}

On the relationship between intelligence and creativity, one classic theory that has persisted in the literature is that intelligence is necessary but not sufficient for creativity (Guilford, 1967; Mackinnon, 1962). The notions of necessity and sufficiency are wellunderstood in classical logic (Hardegree, 1999). Put in binary terms, as intelligence is necessary, an unintelligent person cannot be creative; but as intelligence is not sufficient, an intelligent person may be creative or uncreative.

Beyond binary data, scholars have agreed that such a relationship is best characterized by a graphical observation: Guilford (1967) found that the IQ vs. creativity scatterplot exhibited, roughly, the shape of a right triangle, particularly one whose right angle rests on the bottom right corner (p. 168); and subsequently, researchers (e.g. Karwowski et al., 2016) have effectively treated this shape of scatter as a graphical restatement of the logical theory. See Figure 1 for an example. ${ }^{1}$ So to speak, the theorized relationship then is a two-sided coin: one side logical, in terms of necessity and sufficiency; and the other side graphical, in terms of the triangular shape of the bivariate scatter.

A problem arises from the fact that statistical inference methods available to psychologists, consisting primarily of linear techniques of correlation and regression, are inherently inappropriate for the theorized relationship (Dul, 2016). Thus, to substantiate the classic theory, a more specialized analytic treatment is needed. Such a treatment must satisfy two desiderata: first, it must facilitate formal statistical inference; and second, it must respect the dual nature of the theorized relationship, comprehensively tying together the relevant logical notions to the graphical evidence. Attempts in the literature to provide an appropriate analytic treatment have come in two classes: average-based methods (e.g. Jauk, Benedek, Dunst, \& Neubauer, 2013b) and Necessary Condition Analysis (NCA) (Dul, 2016; Karwowski et al., 2016).

\section{Average-based methods}

Early attempts to analyze the relationship between intelligence and creativity shared a common program, to wit (Guilford \& Christensen, 1973; Hasan \& Butcher, 1966; Yamamoto \& Chimbidis, 1966): the sample was split at a particular threshold, say 120 IQ points; the IQ vs. creativity correlations were compared between the below-threshold and above-threshold subsamples; and the result of the correlation being more positive for the below-threshold group was taken as evidence for the theory. The idea of a specific value of IQ serving as a threshold for a difference in correlations came to be known as the threshold hypothesis, which has become prominent in the literature on intelligence and creativity (for a review, see Jauk et al., 2013b; Karwowski \& Gralewski, 2013).

\footnotetext{
${ }^{1}$ Strictly speaking, the scatter depicted in Guilford (1967, p. 168) was not a right triangle. Furthermore, Dul (2016) pointed out that the "triangular" shape itself can be seen as a special case of a more general "pentagonal" shape still compatible with the necessary-but-not-sufficient interpretation. For instance, the diagonal line drawn on Figure 5 intersects the top edge and left edge of its scatterplot, thus tracing a pentagonal shape. Regardless of terminology, the theorized shape has not been rigorously defined in the literature, a fact we run into in the section "Critique of Necessary Condition Analysis". For pedagogical brevity, we simply call the theorized shape "triangular" or "triangle-shaped" for the remainder of the present article, bearing in mind the ambiguity inherited from the literature.
} 


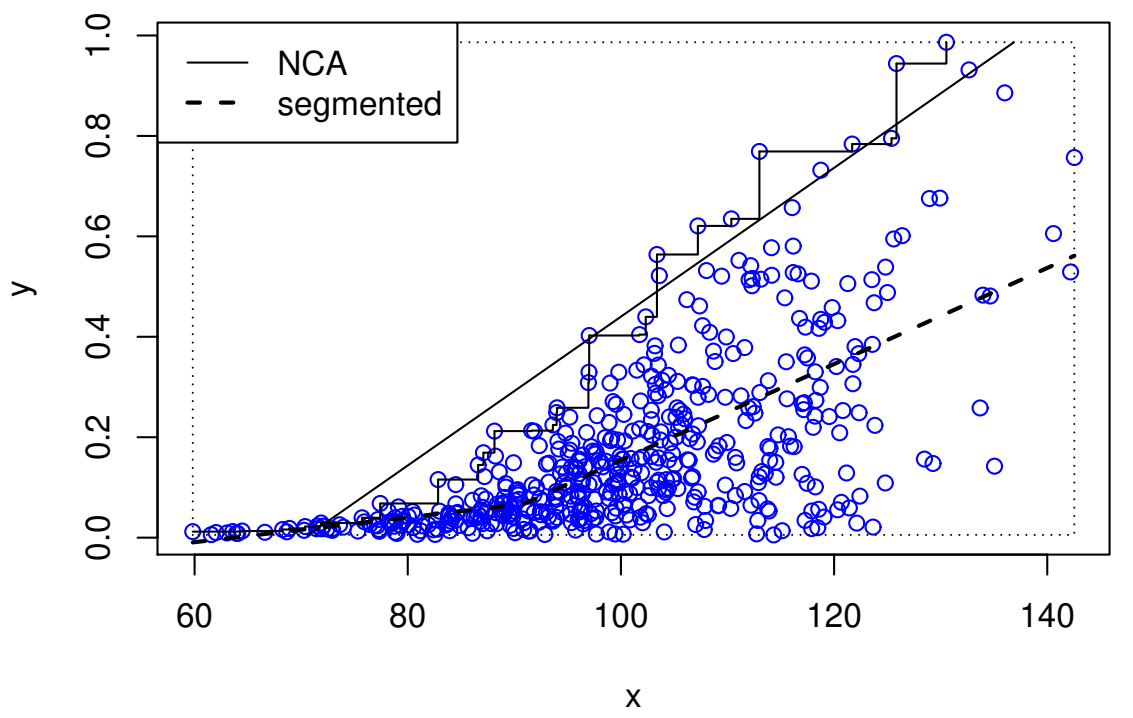

Figure 1. Ideal triangular scatterplot for $X$ as necessary but not sufficient for $Y$, with result from Necessary Condition Analysis (NCA) and segmented regression; $\theta=$ $(.005, .01, .01, .995)$. (See section "Model and hypothesis" for explanation of the parameters.) Solid stepped lines are the NCA ceiling under CE-FDH; solid straight line is the NCA ceiling under CR-FDH. Dashed line is the segmented regression line, with the breakpoint at 90.88 .

The threshold hypothesis was not without fault. A meta-analysis by Kim (2005) showed that support for it was weak. Worse, methodological artifacts raised concern: Sligh, Conners, and Roskos-Ewoldsen (2005) demonstrated that taking subsamples incurred range restriction, affecting threshold-induced correlations; and Karwowski and Gralewski (2013) pointed out that there was no theoretical basis for setting the threshold at any specific value and demonstrated that the value of the threshold obtained in studies depended on methodological choices. More recent attempts (Jauk et al., 2013b; Karwowski, Kaufman, Lebuda, Szumski, \& Firkowska-Mankiewicz, 2017; Shi, Wang, Wang, Zhang, \& Xu, 2017) addressed these issues by employing segmented regression (Muggeo, 2008), a continuous piecewise linear model with one breakpoint, the breakpoint being obtained empirically rather than chosen arbitrarily. See Figure 1 for an example. Such a method allowed testing of the threshold hypothesis while bypassing the objectionable elements of splitting the sample and arbitrarily choosing thresholds. However, segmented regression was not foolproof: even the ideal triangle-shaped scatterplot cannot guarantee that the slope will be smaller to the right of the breakpoint (see Figure 1); not even that a significant breakpoint will be found (see Figure 1d in Jauk et al., 2013b). It is also important to note that having a sharp change in the conditional relationship via a threshold runs contrary to the smooth change in the ideal scatterplot (Guilford \& Christensen, 1973) - it is just as difficult to argue for the existence 
of a hard threshold as to point where it might be.

Beyond thresholds, other attempts at an analytic treatment involved escaping linearity by adding quadratic terms to the regression model (Jauk et al., 2013b; Karwowski \& Gralewski, 2013; Runco, 2014). In this case, a significant quadratic term was taken as evidence for the necessary-but-not-sufficient relationship. Latent trait modeling was also used when comparing correlations (Karwowski \& Gralewski, 2013).

That turning to thresholds, quadratic terms, and latent traits could retain the statistical inference of linear regression (Jauk et al., 2013b; Karwowski \& Gralewski, 2013) satisfied the first desideratum. However, these different methods so far shared a fundamental flaw: they constrained researchers' focus to single point-predictions without regard to the heteroskedasticity inherent in the ideal triangle-shaped scatterplot (Dul, 2016; Runco, 2014). Karwowski et al. (2016) called these methods "average-based" and pointed out their inappropriateness:

Correlation, regression, segmented regression, or even latent variable analysis are not fit to test whether or not one construct may be seen as a necessary condition of another. All these methods, no matter whether they are based on simple ordinary-least-squares estimations or on the more elaborate maximum likelihood estimation, deal with average relationships - namely, they estimate the relationship across the distribution of raw scores. Thus, by definition, they test a different type of relationships. (p. 114)

The second desideratum is where average-based methods fail. Broadly speaking, averagebased methods are an oversimplification in that they attempt to confirm the classic theory by merely confirming a specific feature presumed to be symptomatic of the ideal triangular scatter. Drawing an analogy from the cognitive biases literature, average-based methods can be characterized as an attribute substitution (Kahneman, 2011): the harder question, "does the bivariate distribution have the ideal triangle-shaped scatter?" is implicitly substituted by an easier question, e.g. "is the correlation weaker above 120 IQ than below it?". To the extent that other relevant features are neglected, average-based methods lose the bigger picture and are thus inconclusive.

\section{Necessary Condition Analysis}

A more recent development in the pursuit of an analytic treatment came from outside the psychological literature. In organizational research, Dul (2016) observed a pervasive methodological mismatch: researchers turning to conventional correlational and linear regression techniques despite the underlying theories cast in terms of necessary-but-notsufficient relationships. This mismatch happened to mirror the challenge faced by intelligence and creativity researchers trying to pin down the classic theory, all the way down to the ideal triangle-shaped scatterplot. It was in response to this mismatch that Dul introduced NCA, an "analytic approach" (p. 11) for finding "necessary determinants that are not automatically sufficient" (p. 32). In a nutshell, NCA's solution was to identify the necessary-but-not-sufficient relationship by measuring an "empty upper left corner" in the scatterplot, a large effect indicating the targeted relationship. Note that the upper-left corner of Figure 1 has no observations. Clearly, this approach was more on-point to graphical intuitions than anything intelligence and creativity researchers had been doing. 
Karwowski et al. (2016) innovated the use of NCA on intelligence vs. creativity data, analyzing a large pool of datasets, including several of those in Jauk et al. (2013b), and enthusiastically attested to the utility of NCA for substantiating the classic theory. Subsequently, researchers (Karwowski et al., 2017; Shi et al., 2017) used NCA and segmented regression together. In such studies, researchers by use of NCA have consistently ruled in favor of the necessary-but-not-sufficient relationship. The implication is a consensus that if the data graphically exhibit the ideal triangular scatter, NCA can be relied on to confirm so.

NCA is a burgeoning enterprise in social research. Beyond intelligence and creativity, NCA has been used to examine in terms of necessity the following phenomena: sales as predicted by ambition, sociability, interpersonal sensitivity, and learning approach (Dul, 2016); individualism and innovation (Dul, 2016); workplace spirituality and well-being (Garg, 2017); safety consciousness and productive truck driving (de Vries, de Koster, Rijsdijk, \& Roy, 2017); and metacognition deficits and motivation in people with schizophrenia (Luther et al., 2017).

At first glance, NCA provides the sought-after analytical treatment substantiating the role of intelligence as necessary-but-not-sufficient for creativity. However, as we argue in the present article, a more rigorous assessment of NCA and its use in intelligence vs. creativity data would reveal that it in fact fails to satisfy the desiderata of an analytical treatment. Continued use of NCA for the same problem (Karwowski et al., 2017; Shi et al., 2017 ) is then counterproductive, as doing so gives the false impression that the theorized relationship has been adequately accounted for.

\section{Objectives}

Our objective in the present article is threefold. First, we argue that NCA is not a proper analytic treatment of the necessary-but-not-sufficient relationship. Second, for particularly the scenario of double-bounded (i.e. having both a highest possible value and a lowest possible value) creativity variables, we offer an alternative methodology, in the form of a probability model of creativity as a function of intelligence. Third, we demonstrate the proposed model by reanalyzing applicable data (Jauk et al., 2013a) from Jauk et al. (2013b). Note that the present article has a bent more methodological than substantive - we gloss over minutiae of psychological theory, such as distinctions between creative potential and creative achievement, in favor of focusing on the statistical and logical aspect of the problem, which is how to adequately analytically substantiate the theorized relationship.

We do not seek our proposal to become the official methodology, so to speak, for analyzing the relationship between intelligence and creativity. Instead, we intend merely to re-start the conversation and set the tone by pointing to aspects of the matter that the literature has not adequately addressed. Our own proposal is best seen as a proof of concept rather than an authoritative prescription. Note that while necessary-but-not-sufficient relationships, triangular distributions, and NCA might be relevant in other contexts (e.g. Rosenberg, Knuppe, \& Braumoeller, 2017), our attention in the present article is restricted to intelligence and creativity. Relevant issues in methodology are taken up only in the service of intelligence and creativity, and application to other contexts is beyond the scope of the present article. 
Before we proceed, a note on notation is in order. For pedagogical brevity in the present article, we use $X$ and $Y$ to denote both binary-logic propositions and continuous variables, without disambiguation. On one hand, we trust that wherever the distinction is important, context makes clear which level of measurement applies. For instance, when we point to a scatterplot, then in that context $X$ and $Y$ are continuous rather than binary. On the other hand, in the first place, an underlying assumption of the literature thus far (e.g. Karwowski et al., 2016) is that logical notions can meaningfully be applied to continuous data. Thus, if our account of necessity and sufficiency is correct, then the distinction is moot.

\section{Overview of Necessary Condition Analysis}

Before an account of the shortcomings of NCA in substantiating the theorized relationship in intelligence vs. creativity data, an overview of its framework and methodology is in order. Note that this account is not exhaustive of NCA-it omits concepts not invoked in applications to the intelligence and creativity literature (Karwowski et al., 2016, 2017; Shi et al., 2017). Furthermore, note that in the interest of clarity, the presentation here is slightly more formal than in the relevant accounts of NCA - we refer readers to Dul (2016) and Karwowski et al. (2016) for a gentler introduction.

In binary logic, the necessity of $X$ for $Y$ is expressed by the proposition "no $Y$ without $X$ " (Dul, 2016; Karwowski et al., 2016). NCA was proposed with an aim to bring the logic of necessity to data (Dul, 2016). In particular, the goal was to elucidate the role of the predictor $X$ as necessary for the response $Y$. In contrast, conventional regression has been characterized by proponents of NCA (Dul, 2016; Karwowski et al., 2016; van der Valk, Sumo, Dul, \& Schroeder, 2016) as corresponding to the proposition "if $X$ then $Y$ ", which expresses the sufficiency of $X$. Thus, conventional regression is inappropriate for analyzing necessity, for which a different method is needed.

Broadly speaking, NCA leverages intuitions on both the logical side and the graphical side. On the logical side, adapting the convention that 0 is falsity and 1 is truth, $X$ being necessary-but-not-sufficient for $Y$ is expressed by the inequality $Y \leq X$. A natural generalization of this inequality to the continuous realm is $Y \leq f(X)$ for some nondecreasing function $f$ called the ceiling. On the graphical side, a prominent feature of the triangleshaped scatter is an empty upper left corner. It is not difficult to see that the two go hand in hand: the informativeness of the bound $Y \leq f(X)$ operationalizes the degree of necessity of $X$ for $Y$; and likewise, the area of the region empty from the upper left corner of the scatterplot operationalizes the degree of necessity of $X$ for $Y$. Then a statistic that quantifies necessity as such can be called the effect size, denoted $d$.

From such intuitions, NCA operationalizes necessity as follows. The scope, denoted $\mathcal{S}$, is the entire region covered by the scatterplot. For a given ceiling $f$, the scope is partitioned into a region above, called the ceiling zone, and a region below, called the full zone (Dul, 2016). Finally, let A denote an area operator. Then the effect size is given by

$$
d=\frac{\mathrm{A}\{(x, y) \in \mathcal{S}: y>f(x)\}}{\mathrm{A} \mathcal{S}}
$$

i.e. the relative area of the ceiling zone to the scope. Then clearly, $0 \leq d \leq 1$. The larger $d$ is, the more necessary $X$ is for $Y$. 
By framing necessity in terms of an upper bound, NCA avoids the homoskedasticity implicit in average-based methods. Instead, focus is on the part of the scatterplot where there are no observations (Dul, 2016). In a sense, the role of $X$ as necessary is represented by the inequality $Y \leq f(X)$, analogous to how simple linear regression is represented by the equation $Y=\beta_{0}+\beta_{1} X+\epsilon$ (Dul, 2016). Such an interpretation is compatible with the idea that intelligence imposes an upper limit on creativity (Guilford \& Christensen, 1973; Schubert, 1973). ${ }^{2}$

The choice of ceiling and scope, on which $d$ depends, is nontrivial. Note that if $f$ and $\mathcal{S}$ were set a priori, $d$ would be independent of the data. The NCA way is to choose both empirically, and Dul (2016) provides techniques to do so. Let $\left(x_{1}, y_{1}\right), \ldots,\left(x_{n}, y_{n}\right)$ be the sample observations. For the scope, there is only one technique,

$$
\mathcal{S}=\left\{(x, y) \in \mathbb{R}^{2}: \min _{i} x_{i} \leq x \leq \max _{i} x_{i}, \min _{i} y_{i} \leq y \leq \max _{i} y_{i}\right\}
$$

i.e. set the scope as the smallest rectangle that encloses all the sample observations. For the ceiling, offered are several techniques, in consideration of the informativeness of the bound and the accuracy, the proportion of sample observations below the ceiling. In general, it is desired that the bound is informative and is not inaccurate. In the technique Ceiling Envelopment with Free Disposal Hull (CE-FDH) (Dul, 2016),

$$
f_{\mathrm{CE}-\mathrm{FDH}}(x)=\max \left\{y_{i}: x_{i} \leq x\right\}, \quad \min _{i} x_{i} \leq x \leq \max _{i} x_{i}
$$

i.e. the ceiling is the empirical cumulative maximum. CE-FDH maximizes $d$ under the constraint of perfect accuracy - for a proof, see the Appendix. Another technique, Ceiling Regression with Free Disposal Hull (CR-FDH) (Dul, 2016), smooths the CE-FDH ceiling by taking an OLS regression line over the sample points where CE-FDH has a jump. CRFDH allows observations above the ceiling, making it less sensitive to outliers at the cost of accuracy. See Figure 1, Figure 2, Figure 3, and Figure 4 for examples of CE-FDH and CR-FDH shown graphically. More techniques were proposed in Dul, including quantile regression and stochastic frontier analysis; but only CE-FDH and CR-FDH have been used in applications to intelligence and creativity (Karwowski et al., 2016, 2017; Shi et al., 2017).

NCA provides no formal statistical significance test. Instead, regardless of ceiling technique chosen, Dul (2016, p. 29) prescribed $d>0.1$ as a decision rule for concluding that the empty upper left corner is "large enough to be taken seriously". This decision rule has been used in analyses of intelligence vs. creativity data to conclude support for the role of intelligence as necessary-but-not-sufficient (Karwowski et al., 2016, 2017; Shi et al., 2017). In contrast to the threshold hypothesis, the theory as substantiated by NCA is termed necessary condition hypothesis (Karwowski et al., 2016).

\section{Critique of Necessary Condition Analysis}

At first glance, it is in NCA that research finally converges to a method that successfully confirms the theorized relationship between intelligence and creativity. Of course,

\footnotetext{
${ }^{2}$ Outside the context of intelligence and creativity, the imposed-upper-limit interpretation is hinted by Wilkinson and the Task Force on Statistical Inference (1999, p. 597), who report a triangular scatterplot between a participant's age and their "number of years together in current relationship" —of course, a person cannot have been in a relationship for longer than they have been alive.
} 
such success would entail that NCA satisfies the two desiderata. To the contrary, we argue that NCA satisfies neither desideratum. On the first, NCA lacks apparatus for probability and statistical inference; and on the second, NCA fails to account for key features of the theorized relationship. Thus, conclusions yielded by NCA in support of the theorized relationship (Karwowski et al., 2016, 2017; Shi et al., 2017) were premature, and providing an analytical treatment for the theorized relationship remains an open problem.

\section{Failing to satisfy the first desideratum}

Conventional statistical methods, such as linear regression, are formal in that they involve statistical inference. In statistical inference, the conclusion depends not only on the value of the statistic obtained from the sample at hand but also on the sampling distribution of this statistic. Such a setup can be as involved as formally specifying a probability model for the data-generating process then deriving the distribution of the statistic from this model (Casella \& Berger, 2002). The setup can also be as cursory as using a resampling method to produce an empirical sampling distribution (Neal \& Simons, 2007). In any case, probability theory is leveraged so as to account for sampling error, and doing so sets apart an inferential method from a descriptive one.

In contrast, NCA studies of intelligence and creativity (Karwowski et al., 2016, 2017; Shi et al., 2017) missed opportunities to incorporate the desired formalism. Specifying a probability model from the ground up is understandably difficult. But barring that, NCA researchers could have turned to quantile regression, considered by Dul (2016) to be a viable ceiling technique - doing so could have provided for the probabilistic interpretation hinted at by Karwowski et al. (2017) of the ceiling zone having low but nonzero probability. And barring that, NCA researchers could have turned to resampling methods, a possibility raised also in Dul. Instead, NCA researchers have preferred CE-FDH and CR-FDH, which are not specified to provide probability statements, and have arrived at a verdict without accounting for sampling error. Apparent from these preferences is a misguided expectation of conventional formalism as an output amid a failure to follow through with the requisite inputs.

Such contrast is remarkable in light of its two misleading implications. First, eschewing regression on the grounds of homoskedasticity, as Karwowski et al. (2016) did, seems to imply that regression is exclusively homoskedastic. But regression encompasses a wide realm of possibilities, of which some are heteroskedastic if specified to be so. For instance, logistic regression, which models the response $Y$ as a coin flip whose success probability depends on predictor $X$ is heteroskedastic - a fair coin has more variance than one with, say, $99 \%$ probability of heads. Second, amid its lack of apparatus for proper statistical inference, endorsing NCA as confirming the theorized relationship seems to imply that statistical inference is dispensable. But in the context of the problem, statistical inference is the goal toward which an analytical treatment is applied. If statistical inference were unimportant, then to substantiate the theory, it would have been enough to simply point to the triangular scatterplot in the first place - which, in the first place, had already been done as early as Guilford (1967).

Simply put, the rationale behind NCA erroneously conflates homoskedastic linearity and formalism; and endorsing NCA, rather than offering a solution, sweeps the problem under the rug. 


\section{Failing to satisfy the second desideratum}

Keeping with the logic motif, NCA boils down to an argument, one where the premise is "there is a large-enough empty upper left corner" and the conclusion is "the theorized relationship holds". In that sense, the validity of this argument is equivalent to satisfaction of the second desideratum. To demonstrate NCA's inadequacy as an operationalization of the theorized relationship, we present three counterexamples to this argument. These counterexamples are scenarios of bivariate distributions for which the premise is true but the conclusion is either patently false or ambiguous at best. We break down each counterexample to explicate what NCA fails to account for, and this elaboration doubles as a guideline for what it would take for an analytical treatment to satisfy the second desideratum.

Preliminary to these counterexamples, some notes on corners and bounds are in order. As can be gleaned from the NCA definitions of effect size $d$ and ceiling $f$, leveraged by $\mathrm{NCA}$ is an intuition that an empty upper left corner can be recast as a nondecreasing upper bound. Towards a more comprehensive account of necessity and sufficiency, we find it useful to extend this intuition to a more general mapping between empty corners and monotonic bounds, to wit: an empty upper left corner is to a nondecreasing upper bound; an empty upper right corner is to a nonincreasing upper bound; an empty lower left corner is to a nonincreasing lower bound; and an empty lower right corner is to a nondecreasing lower bound. Whereas the language in NCA works (Dul, 2016; Karwowski et al., 2016) is in terms of empty corners, we find it useful to speak in terms of bounds. We present the counterexamples graphically, so for pedagogical brevity, we invoke notions of "upper bound" and "lower bound" only casually - it should be enough to say that NCA-like intuitions should work well and that we trust the reader to eyeball-estimate each bound as it is verbally pointed to.

First counterexample Figure 2 prototypes a family of scenarios of which each could be called, for the most part, a positive linear relationship. For the data in Figure 2, CE-FDH $d=0.406$, a positive result towards the conclusion that $X$ is necessary but not sufficient for $Y$, which is obviously untenable. On one hand to NCA's credit, $X$ is indeed necessary, as "no $Y$ without $X$ " holds true in the scatterplot. On the other hand, the bivariate scatter obtained is far from the ideal triangular scatter characteristic of the theorized relationship. Necessity is accounted for; yet the theorized relationship is not obtained.

To make sense of the first counterexample, realize that necessity and sufficiency are independent. The relationship in this counterexample is best described by the conditional proposition " $X$ is necessary and sufficient for $Y$ " - both necessity role "no $Y$ without $X$ " and sufficiency role "if $X$ then $Y$ " hold true in the scatterplot. On one hand, NCA is half-correct in that it confirms the necessity of $X$ via a nondecreasing upper bound. On the other hand, $\mathrm{NCA}$ is half-wrong in that it fails to account for the nondecreasing lower bound, which would have ruled out the theorized relationship. From there, it is easy to see that just as a nondecreasing upper bound is indicative of necessity, neglected is that a nondecreasing lower bound is indicative of sufficiency. In short, the necessary condition hypothesis (Karwowski et al., 2016) is not the necessary-but-not-sufficient hypothesis. Put in another way, NCA's fundamental error is in its mapping of logical propositions to scatterplots. Recall that 


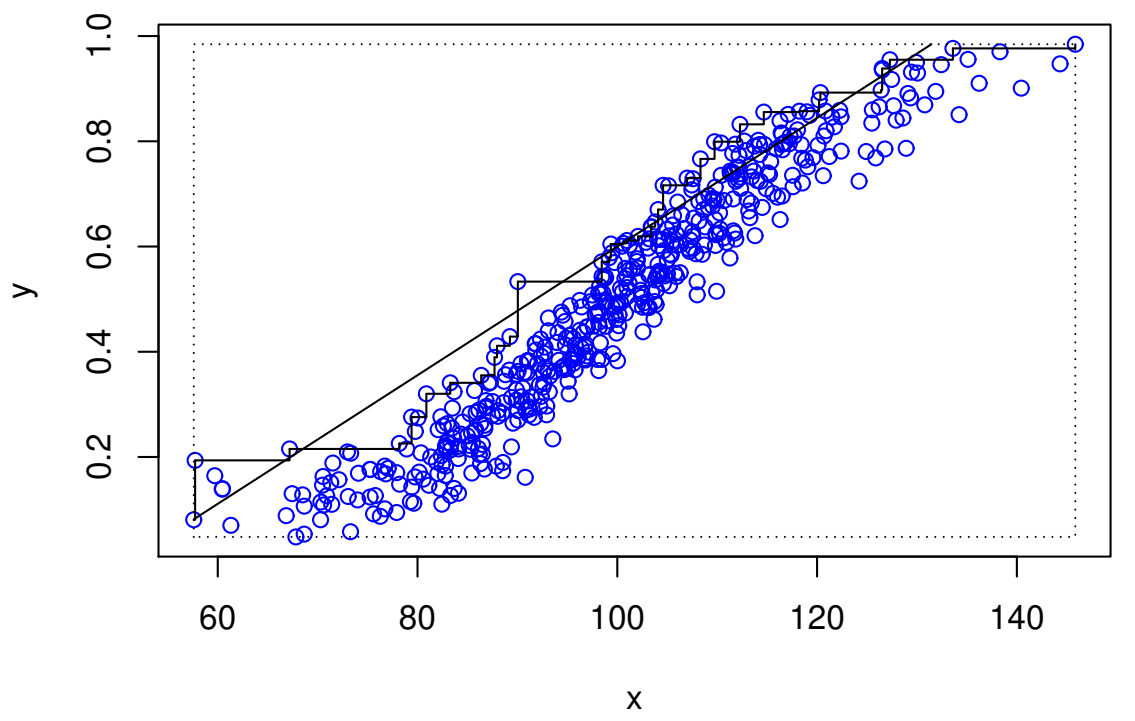

Figure 2. Counterexample scenario of positive linear scatterplot, with result from Necessary Condition Analysis (NCA); $\theta=(.005, .3, .7, .995)$. Stepped lines are the NCA ceiling under $\mathrm{CE}-\mathrm{FDH}$; straight line is the NCA ceiling under CR-FDH.

NCA's mapping is, to wit (Dul, 2016; Karwowski et al., 2016): " $X$ is necessary for $Y$ " is to the ideal triangular scatterplot; and " $X$ is sufficient for $Y$ " is to the positive linear scatterplot. In contrary, the correct mapping is, to wit: " $X$ is necessary but not sufficient for $Y$ " is to specifically the ideal triangular scatterplot; " $X$ is necessary and sufficient for $Y$ " is to specifically the positive linear scatterplot; and " $X$ is necessary for $Y$ " is to an abstraction under which the ideal triangular scatterplot and positive linear scatterplot are special cases. Thus, correct methodology must break the necessary-but-not-sufficient proposition down to its necessity and sufficiency parts and treat them separately.

Noting that linear regression works well for the first counterexample, the characterization of linear regression as catering exclusively to sufficiency (Karwowski et al., 2016; van der Valk et al., 2016) is also fallacious. A strong positive correlation evidences the conditional proposition " $X$ is necessary and sufficient for $Y$ ", while a strong negative correlation evidences the opposite, " $X$ is necessary and sufficient for not- $Y$ ". Thus, rather than dealing exclusively with sufficiency, linear regression conflates necessity and sufficiency together. Interestingly, if $Y$ is bounded from above and bounded from below by the same quantity, an equality is obtained, and in that sense, average-based results are within the purview of necessity and sufficiency.

Second counterexample Figure 3 prototypes a family of scenarios where the shape of the scatter is that of a right-opening megaphone. For the data in Figure 3, CE-FDH $d=$ 0.217 , again an untenable positive result. There is a nondecreasing upper bound, but there is also a nonincreasing lower bound. Whereas NCA identifies a nondecreasing 


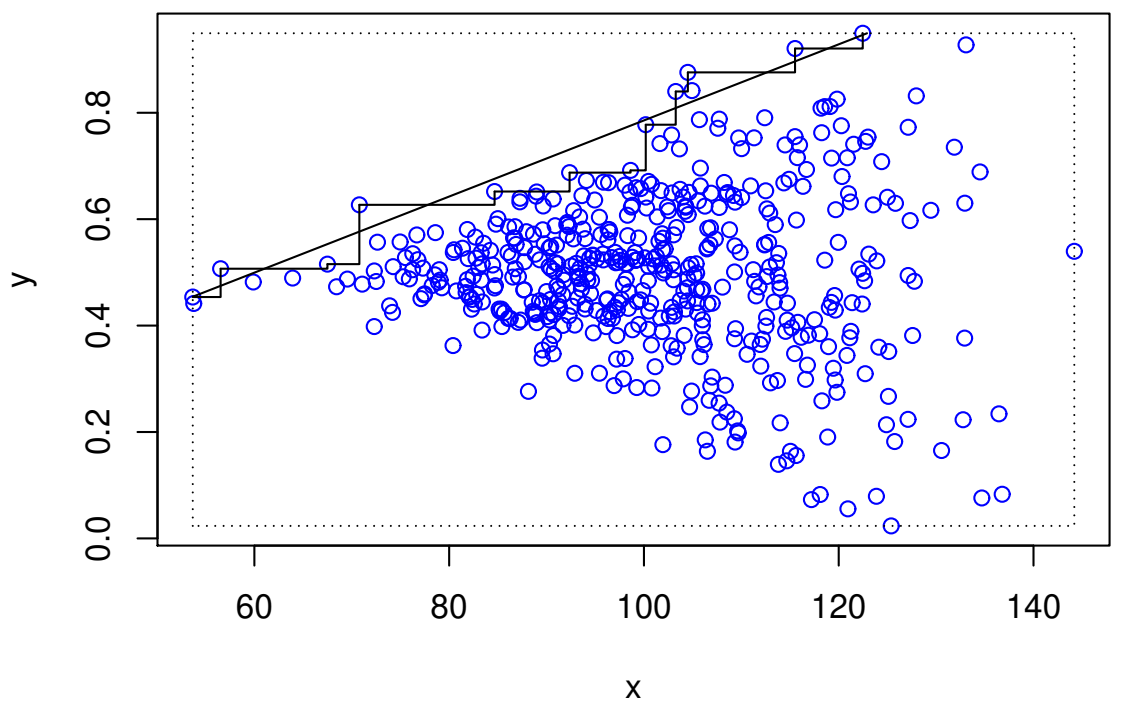

Figure 3. Counterexample scenario of right-opening megaphone scatterplot, with result from Necessary Condition Analysis (NCA); $\theta=(.4, .6, .005, .995)$. Stepped lines are the NCA ceiling under CE-FDH; straight line is the NCA ceiling under CR-FDH.

upper bound with the conditional proposition " $X$ is necessary for $Y$ ", the shape of the bivariate scatter seems to pose a challenge to any mapping between conditional propositions and monotonic bounds. On one hand, taking the nondecreasing upper bound in isolation - as we already do for the ideal triangular scatter and the positive linear relationship - should indicate that $X$ is necessary for $Y$. On the other hand, at an intuitive level, the shape of the bivariate scatter is difficult to parse in terms of necessity and sufficiency, as if it were intractable in those terms.

To make sense of the second counterexample, the apparent lack of necessity and sufficiency in the relationship can be framed in terms of opposites cancelling out. To illustrate in a more familiar context, recall that in the sample correlation coefficient, the numerator $\sum_{i=1}^{n}\left(x_{i}-\bar{x}\right)\left(y_{i}-\bar{y}\right)$ is, in a sense, the net signed evidence to the linear relationship. In this sense, the $i$ th sample observation evidences the positive linear relationship if its product of deviations $\left(x_{i}-\bar{x}\right)\left(y_{i}-\bar{y}\right)$ is positive, by that magnitude, but by the same magnitude evidences the negative relationship if the same product is negative. Furthermore, if the sample correlation coefficient is zero, then the evidence is even-handed between the opposite directions - they cancel out, so overall, neither direction is apparent. The same cancel-out mechanics can be applied analogously to necessity and sufficiency. Whereas correlation has the product of deviations, analogous to necessity and sufficiency is the informativeness of the monotonic bound. If the bounds were linear, as is the case for this counterexample, a bound's informativeness could be quantified by its slope magnitude. In terms of necessity, whereas the nondecreasing upper bound evidences the conditional proposition " $X$ is 
necessary for $Y$ ", it can be seen that the nonincreasing lower bound evidences an opposite conditional proposition, "not- $X$ is necessary for $Y$ ". In the case of this counterexample, the bounds' slope magnitudes are equally large, so the corresponding logical propositions cancel out, and overall, neither necessity role is apparent. In terms of sufficiency, whereas the nondecreasing upper bound evidences the conditional proposition "not- $X$ is sufficient for not- $Y$ ", the nonincreasing lower bound evidences an opposite conditional proposition, "not- $X$ is sufficient for $Y$ ". Again, there is cancelling out, so overall, neither sufficiency role is apparent. Either way, since the cancel-out is due to two sides of bound having opposite monotonicities, we call it a between-sides cancel-out. Thus, by cancel-out mechanics, the strategy of associating monotonic bounds with conditional propositions remains sound.

It turns out that cancel-out mechanics also provides insight towards properly dealing with the denial of a role, as in " $X$ is not sufficient for $Y$ ". Again turning to correlations, to deny " $X$ is correlated to $Y$ " is ambiguous: to deny specifically the positive linear relationship is to say $\operatorname{Corr}(X, Y) \leq 0$, which is to say that the positive linearity does not exceed the negative linearity; while to deny a linear relationship in either direction is to say $\operatorname{Corr}(X, Y)=0$, which is to say that the positive linearity and the negative linearity cancel out. However, when we make this denial, we usually mean the latter rather than the former. Analogously for conditional relationships, when we say " $X$ is not sufficient for $Y$ ", it must be made clear that we do not mean to assert the proposition "not- $X$ is sufficient for $Y$ "-instead, we mean that the opposite propositions " $X$ is sufficient for (unreversed) $Y$ " and " $X$ is sufficient for not- $Y$ " cancel out. Whereas " $X$ is sufficient for $Y$ " is evidenced by a nondecreasing lower bound, " $X$ is not sufficient for $Y$ " requires that the lower bound be neither increasing nor decreasing - which is what would be seen in the ideal triangular scatterplot. In that case, since the cancel-out is due to one side of bound being constant, we call it a within-side cancel-out.

Third counterexample Figure 4 prototypes a family of scenarios where both upper bound and lower bound are increasing but at different rates. For the data in Figure 4 , CE-FDH $d=0.426$, a positive result. However, it is unclear whether such a scatterplot truly counts as evidence in favor of the theorized relationship. There is a prominent empty upper left corner, which evidences the necessity of $X$ for $Y$; but there is also a less prominent empty lower right corner, which evidences the sufficiency of $X$ for $Y$. The empty lower right corner is too large for the ideal triangular scatterplot yet too small for the positive linear relationship. Thus, the scatterplot falls under a grey area between unequivocal confirmation and unequivocal disconfirmation of the theorized relationship.

The third counterexample demonstrates that the theorized relationship is only vaguely represented. Pointing at select triangular scatterplots, as has been the practice in the literature (Guilford, 1967; Guilford \& Christensen, 1973; Karwowski et al., 2016), has not adequately answered the question of how far our tolerance must be for less-than-perfect triangular scatterplots. In fact, the schematic triangular scatterplot in Guilford (1967, p. 168) was itself less than perfect-instead of lying parallel to the horizontal axis, the lower bound rose perceptibly though not as pronounced as the upper bound did. What is clear, at least, is that the upper bound must be more informative than the lower bound; 


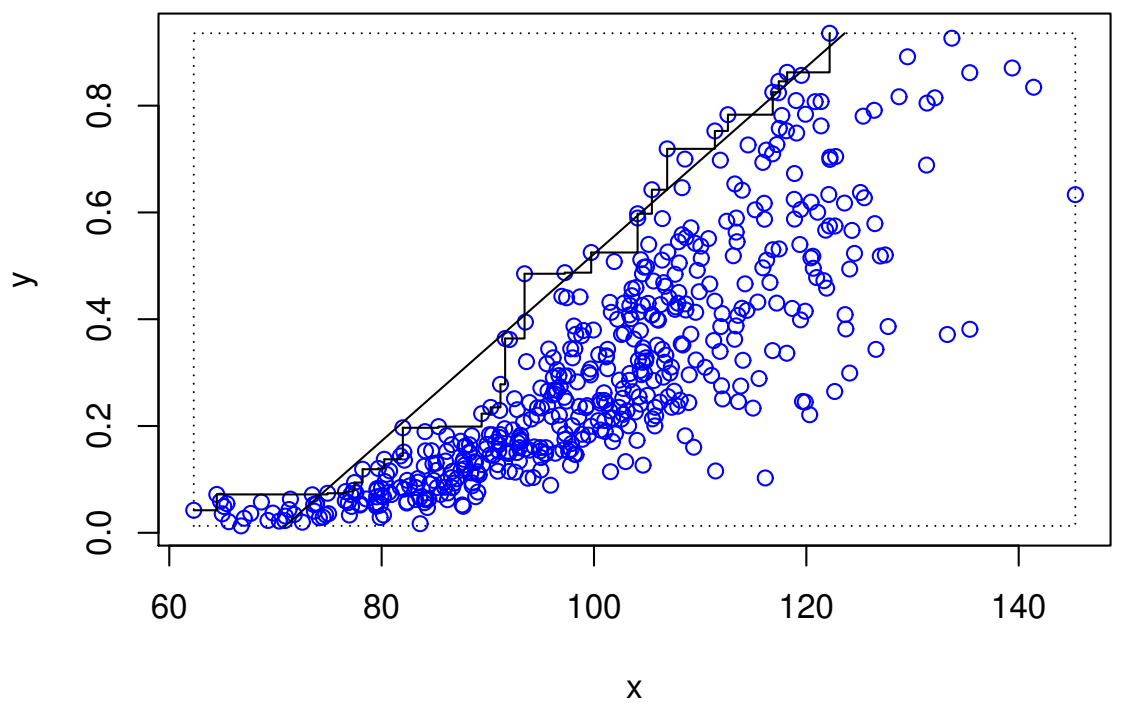

Figure 4. Counterexample scenario of increasing both upper bound and lower bound but at different rates, with result from Necessary Condition Analysis (NCA); $\theta=$ $(.005, .1, .15, .995)$. Stepped lines are the NCA ceiling under CE-FDH; straight line is the NCA ceiling under CR-FDH.

otherwise, the scenario would be closer to " $X$ is sufficient but not necessary for $Y$ " than the theorized relationship.

What these three counterexamples make clear is that, just like average-based methods, $\mathrm{NCA}$ is a faulty attribute substitution - answering the easier question "is there a largeenough empty upper left corner?" does not answer the target question "does the bivariate distribution have the ideal triangle-shaped scatter?". Each counterexample shows what else needs answered on the way to determining whether or not the theorized relationship is confirmed. Putting together these three, one overarching lesson can be learned: to comprehensively tie together logical notions and graphical evidence, an analytical treatment must account for not only both sides (i.e. upper or lower) of bound but also their respective monotonicities (i.e. nondecreasing, constant, or nonincreasing) and respective measures of informativeness. In the case of linear bounds, the monotonicity is indicated by the slope sign (i.e. positive, zero, or negative) while the informativeness is quantified by the slope magnitude.

\section{Model and hypothesis}

We endeavor to provide a proper analytical treatment substantiating the theorized relationship between intelligence and creativity. In light of the two desiderata and NCA's pitfalls, this analytical treatment must neither fall in the trap of conflating homoskedastic linearity and probability theory nor fall in the trap of oversimplified attribute substitutions. 
For the present article, we provide an analytical treatment particularly for a continuous and double-bounded measure of creativity as a function of IQ. Such a scenario applies to some creativity scores (e.g. Jauk et al., 2013a) that are from a self-report inventory or are mean Likert ratings.

We present our proposed analytical treatment in two stages. First, we posit a probability model, parametrized by $\theta$, that accounts for necessity and sufficiency between IQ and creativity regardless of whether the theorized relationship is true or false. Second, we make the theorized relationship falsifiable by formally restating it as a hypothesis (i.e. the alternative hypothesis) in terms of $\theta$. If, for the population of IQ and creativity scores, the hypothesis is true of $\theta$, then the theorized relationship is true; otherwise, the theorized relationship is false.

\section{Model}

Towards a model for necessity and sufficiency in IQ vs. creativity data, the following strategy is in order. Like NCA, the bivariate relationship is cast in terms of a monotonic ceiling function; but unlike NCA, a monotonic floor function is accounted for as well. Unlike NCA, the ceiling and floor are explicitly specified as soft bounds such that they enclose a region with a pre-specified probability - such is done by specifying a conditional distribution for which the prediction interval with the same probability has an upper limit determined by the ceiling and a lower limit determined by the floor.

Formally, let $0 \leq Y \leq 1$ be the response, the creativity score; and let $X \in \mathbb{R}$ be the predictor, IQ. Then we propose a model as follows.

Model. Let $F^{-1}(p ; \alpha, \beta)$ be the $p$ quantile of a beta distribution with shape parameters $\alpha$ and $\beta$, and let $U=\Phi\left(\frac{X-100}{15}\right)$. Then $Y \mid\{X=x\} \sim \operatorname{beta}\left(\alpha_{x}, \beta_{x}\right)$ where

$$
\begin{aligned}
& F^{-1}\left(0.995 ; \alpha_{x}, \beta_{x}\right)=b_{0}+\left(b_{1}-b_{0}\right) u \\
& F^{-1}\left(0.005 ; \alpha_{x}, \beta_{x}\right)=a_{0}+\left(a_{1}-a_{0}\right) u
\end{aligned}
$$

and

$$
\begin{aligned}
& 0<a_{0}<b_{0}<1 \\
& 0<a_{1}<b_{1}<1 .
\end{aligned}
$$

In the model proposed, details were filled in via a few simplifying assumptions: the probability of the enclosed region being fixed at $99 \%$, consequently making the ceiling the 0.995 quantile and the floor the 0.005 quantile; the ceiling and the floor being linear functions; and the conditional distribution being beta-distributed. On the linearity of the ceiling and the floor, such a choice affords slope signs and slope magnitudes being easily read off the parameters, as well as the overall shape of the bivariate scatter being easily visualized. We call $b_{1}-b_{0}$ the ceiling slope and $a_{1}-a_{0}$ the floor slope. As the IQ score $X$ is itself not double-bounded, the linearity is in its quantile rank $U{ }^{3}$ On the conditional distribution being beta-distributed, such a choice leverages a useful property of the beta distribution: that a specific beta distribution can be identified with only two quantiles (Van

\footnotetext{
${ }^{3}$ By construction, IQ is normal-distributed with mean 100 and standard deviation 15 (Hunt, 2011). Then it follows that $U=\Phi\left(\frac{X-100}{15}\right)$ is the quantile rank of $X$.
} 


\begin{tabular}{|c|c|}
\hline $\begin{array}{l}b_{1}-b_{0}>0 \\
\text { LP: " } X \text { is necessary for } Y " \\
\text { CB: increasing upper bound } \\
\text { EC: empty upper left corner }\end{array}$ & $\begin{array}{l}b_{1}-b_{0}<0 \\
\mathbf{L P}: \text { "not- } X \text { is necessary for } Y \text { "; " } X \text { is } \\
\text { sufficient for not- } Y \text { " } \\
\text { CB: decreasing upper bound } \\
\text { EC: empty upper right corner }\end{array}$ \\
\hline $\begin{array}{l}a_{1}-a_{0}<0 \\
\text { LP: "not- } X \text { is sufficient for } Y \text { "; " } X \text { is } \\
\text { necessary for not- } Y \text { " } \\
\text { CB: decreasing lower bound } \\
\text { EC: empty lower left corner }\end{array}$ & $\begin{array}{l}a_{1}-a_{0}>0 \\
\mathbf{L P}: \text { : } X \text { is sufficient for } Y \text { " } \\
\text { CB: increasing lower bound } \\
\text { EC: empty lower right corner }\end{array}$ \\
\hline
\end{tabular}

Table 1

Summary of interpretation for the model proposed, in terms of logical proposition $(L P)$, continuous bound (CB), and empty corner (EC). Each cell corresponds to a combination of side (i.e. ceiling or floor) and sign (i.e. positive or negative) of slope. The cells are arranged such that each cell's location is analogous to the graphical location of the empty corner in the scatterplot.

Dorp \& Mazzuchi, 2000). ${ }^{4}$ Note that these simplifying assumptions are solely to make the treatment of necessity and sufficiency tractable - other specifications in line with necessity and sufficiency may be possible but more cumbersome. ${ }^{5}$

To demonstrate the model proposed, let $\theta=\left(a_{0}, b_{0}, a_{1}, b_{1}\right)$. Figure 1, Figure 2, Figure 3, and Figure 4 are in fact simulations of the model, 500 observations each: $\theta=(.005, .01, .01, .995)$ in Figure $1 ; \theta=(.005, .3, .7, .995)$ in Figure $2 ; \theta=(.4, .6, .005, .995)$ in Figure 3 ; and $\theta=(.005, .1, .15, .995)$ in Figure 4 . As intended, the model covers both the ideal triangular scatter and the NCA counterexamples.

Accounting for necessity and sufficiency involves navigating through a formidable mix of logical propositions, continuous bounds, and empty corners. Table 1 consolidates the relevant mappings. For instance, the case $b_{1}-b_{0}>0$ is read, to wit: in terms of logical propositions, " $X$ is necessary for $Y$ "; in terms of continuous bounds, an increasing upper bound; and in terms of empty corners, an empty upper left corner. The rest of the cases are read likewise. As should be the case, equivalent propositions fall under the same case. ${ }^{6}$ Cancel-outs are expressed in terms of the model parameters as well. A within-side cancel-out (e.g. " $X$ is not sufficient for $Y$ " in Figure 1 ) is expressed by a zero slope: a zero-slope ceiling (i.e. $b_{1}-b_{0}=0$ ) falsifies the necessity of $X$ for $Y$; and a zero-slope floor

\footnotetext{
${ }^{4}$ The property as stated here is simplified. To be technically correct, the property from Van Dorp and Mazzuchi (2000) is, to wit: there exists a unique pair of beta shape parameters $(\alpha, \beta)$ such that $Y \sim \operatorname{beta}(\alpha, \beta)$ satisfies both $\mathbb{P}\left\{Y \leq t_{p}\right\}=p$ and $\mathbb{P}\left\{Y \leq t_{q}\right\}=q$, where $0<p<q<1$.

${ }^{5}$ For example, we could have achieved a $99 \%$-probability enclosed area by simply using standard quantile regression (see Cade \& Noon, 2003) to estimate an upper quantile line and a lower quantile line. But estimating quantile regression lines separately may result in them crossing over, and preventing this issue is a complicated matter ( Liu \& Wu, 2011).

${ }^{6}$ In logic, equivalent are the following four conditional propositions (Hardegree, 1999): " $P$ is necessary for $Q$ "; " $Q$ is sufficient for $P$ "; "not- $P$ is sufficient for not- $Q$ "; and "not- $P$ is necessary for not- $Q$ ". With respect to Table 1, three operations obtain a non-equivalent proposition: reverse-coding $P$ obtains the opposite case in the same row; reverse-coding $Q$ obtains the opposite case in the same column; and swapping between "necessary" and "sufficient" goes diagonally across.
} 
(i.e. $a_{1}-a_{0}=0$ ) falsifies the sufficiency of $X$ for $Y$. A between-side cancel-out (e.g. Figure 3 ) is expressed by opposite signs with equal magnitude (i.e. $a_{1}-a_{0}=-\left(b_{1}-b_{0}\right)$ ). In Table 1 , a within-side cancel-out occurs between two cells in the same row, while a between-side cancel-out occurs between two cells in the same column. Also note that if both withinside cancel-outs occur simultaneously (i.e. $a_{1}-a_{0}=b_{1}-b_{0}=0$ ), then $Y$ is marginally beta-distributed and independent of $X$.

The model proposed is in fact a regression model albeit in a broader sense than average-based methods are. Point-predictions are provided by a property of the beta distribution:

$$
\mathbb{E}(Y \mid\{X=x\})=\frac{\alpha_{x}}{\alpha_{x}+\beta_{x}} .
$$

Note that whereas the response in linear regression can be broken down into a deterministic function of $X$ and a random error independent of $X$, there is no analogous decomposition in the model proposed. In linear regression, $X$ merely shifts the distribution of the responsethe centered quantity $Y-\mathbb{E}(Y \mid\{X=x\})$ has the same distribution regardless of $X$, so the

model is homoskedastic. In contrast, in the model proposed, $X$ changes the conditional beta shape parameters $\left(\alpha_{x}, \beta_{x}\right)$ - there is neither an assumption of homoskedasticity nor any sense in speaking in terms of residuals. Consequently, regression diagnostics involving residuals, such as plotting a histogram of residuals, are uninformative. In lieu of a goodnessof-fit test, graphical inspection of simulations from the model is crucial for assessing the appropriateness of the model to the data. After all, theory is substantiated with respect to actual data, not abstract ideas. If the model proposed were far off the actual data-generating process, then our analytical treatment would be an empty promise.

\section{Hypothesis}

Towards a hypothesis in terms of the model parameters, we enumerate features that obtain under the theorized relationship. Clearly, the ceiling slope must be positive. Clearly, the floor slope must be nonnegative: a zero slope obtains a perfect triangular scatterplot (e.g. Figure 1); and if less-than-perfect triangular scatterplots (e.g. Figure 4) are confirmatory, then some positive floor slopes are confirmatory. At the same time, since the positive ceiling slope must be larger than the floor slope, neither a positive ceiling slope nor a positive floor slope guarantees confirmation. Note that as limited by the theorized relationship, these features are stated only vaguely.

To consolidate features of the theorized relationship, one strategy is to impose cutoffs: the floor slope is not too large by virtue of not exceeding some fixed cut-off $c_{a}$ while the ceiling slope is large enough by virtue of exceeding some fixed cut-off $c_{b}$, i.e.

$$
0 \leq a_{1}-a_{0} \leq c_{a} \leq c_{b}<b_{1}-b_{0}<1
$$

which we call a cut-off hypothesis. Note that the case $c_{a}=0$ obtains a hypothesis that accepts only perfect triangular scatterplots. Another strategy is to eschew cut-offs altogether, instead taking the bare minimum of the ceiling slope exceeding the floor slope, i.e.

$$
0 \leq a_{1}-a_{0}<b_{1}-b_{0}
$$


which we call the minimal hypothesis. Note that the minimal hypothesis is a weaker claim, implied by every cut-off hypothesis - if $\theta$ satisfies any cut-off hypothesis, then $\theta$ also satisfies the minimal hypothesis.

For the present article, we prefer the minimal hypothesis to represent the theorized relationship. We find little grounding for choosing any particular $\left(c_{a}, c_{b}\right)$ over others, so the advantage of the minimal hypothesis is that this choice need not be made. The disadvantage is that no intuition of "not too large" for the floor nor of "large enough" for the ceiling is encoded in the hypothesis, so a counterintuitive ruling may result for $\theta$ values that barely satisfy the weaker claim. Thus, it must be made clear that we trade off substantiating a stronger claim in favor of escaping a difficult choice of cut-off. ${ }^{7}$

\section{Data and method}

Using the model proposed, we reanalyze an intelligence vs. creativity dataset published in the literature.

\section{Data}

Data (Jauk et al., 2013a) were from Jauk et al. (2013b), a size $n=297$ sample. $^{8}$ For intelligence, the IQ score was taken. For creativity, taken were two creativity scores, Creative Potential Average Originality (CPA) and Creative Achievement (CA). For CPA, the possible values were integers in the range from 1 to 4 . For $\mathrm{CA}$, the possible values were integers in the range from 0 to 440 (M. Benedek, personal communication, November 21, 2016). Both creativity scores were double-bounded and were taken to be continuous. More details on the variables are in Jauk et al. (2013b). ${ }^{9}$ The fluency variable in Jauk et al. (2013b) was not double-bounded and was thus excluded from reanalysis.

\begin{tabular}{lccccc}
\hline & Mean & SD & Correlation to IQ & Min & Max \\
\hline IQ & 107.21 & 14.63 & +1.00 & 59.27 & 147.37 \\
CPA & 1.82 & 0.12 & +0.35 & 1.45 & 2.16 \\
CA & 40.72 & 35.15 & +0.28 & 0 & 208 \\
\hline
\end{tabular}

Table 2

Descriptive statistics for data to be reanalyzed from Jauk et al. (2013b). CPA = Creative Potential Average; $C A=C r e-$ ative Achivement.

Figure 5 and Figure 6 are the scatterplots of the raw data with the NCA lines drawn. Note that the two scatterplots share the same predictor variable, IQ. Table 2 reports descriptive statistics. Under CE-FDH, NCA effect sizes were $d=0.188$ for CPA and $d=0.389$

\footnotetext{
${ }^{7}$ This trade-off is reminiscent of what methodologists (e.g. Cohen, 1994) complain about zero-difference null hypotheses: researchers forgoing specifying difficult cut-offs so as to be able to say "well, the difference is not zero". The consequence is a divergence between statistical significance and practical significance. Make of our choice what you will.

${ }^{8}$ We gained access to the data by writing the first author of the study.

${ }^{9}$ Four IQ-CA pairs were omitted as outliers in the analysis of Jauk et al. (2013b) but are included here. These observations have CA scores 189 and above and are easily found in the scatterplot.
} 
for CA. For both creativity scores, $d>0.1$, so the NCA result was positive. While an empty upper left corner was apparent in both scatterplots, IQ vs. CPA was arguably less ideal. It should be noted that the perception of empty corners and a triangle shape may depend on the aspect ratio of the plot (see Fink, Haunert, Spoerhase, \& Wolff, 2013). For the present article, we used a 4:3 aspect ratio for all scatterplots.

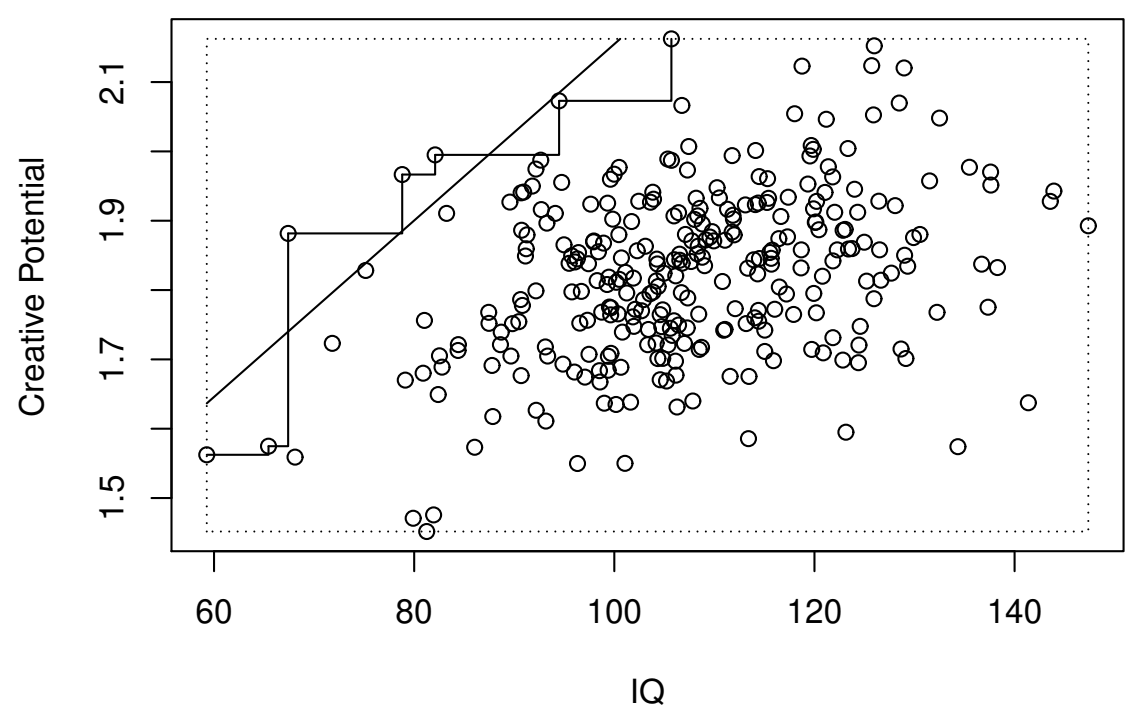

Figure 5. IQ vs. Creative Potential Average Originality (CPA) from Jauk et al. (2013b), with result from Necessary Condition Analysis (NCA). Stepped lines are the NCA ceiling under CE-FDH; straight line is the NCA ceiling under CR-FDH.

\section{Method}

The same procedure was carried out separately for IQ vs. CPA and IQ vs. CA. Prior to analysis, the creativity variable was shifted and scaled to match $0 \leq Y \leq 1$ in the model, i.e. $\frac{\mathrm{CPA}-1}{4-1}$ and $\frac{\mathrm{CA}-0}{440-0}$. The entire analysis was carried out in $\mathrm{R}$ version 3.2.3 (R Core Team, 2015).

As a limitation of the beta distribution, four observations were excluded from the IQ vs. CA analysis due to having a CA score of 0 . Zero scores cause problems in maximum likelihood estimation, as many beta distributions have zero density at $y=0$ and $y=1$.

For parameter estimation, the maximum likelihood estimate, which we denote $\hat{\theta}$, was obtained. As a corollary of the model, $\theta$ maps each $x$ to a pair of quantiles $\left(F^{-1}\left(0.005 ; \alpha_{x}, \beta_{x}\right), F^{-1}\left(0.995 ; \alpha_{x}, \beta_{x}\right)\right)$. Iterative algorithms provided by Van Dorp and Mazzuchi (2000) were used to match this pair of quantiles to the corresponding pair of beta distribution shape parameters $\left(\alpha_{x}, \beta_{x}\right)$. From there, the likelihood function was maximized numerically via the Nelder-Mead simplex with linear constraints as implemented in the $\mathrm{R}$ function stats : : constrOptim. 


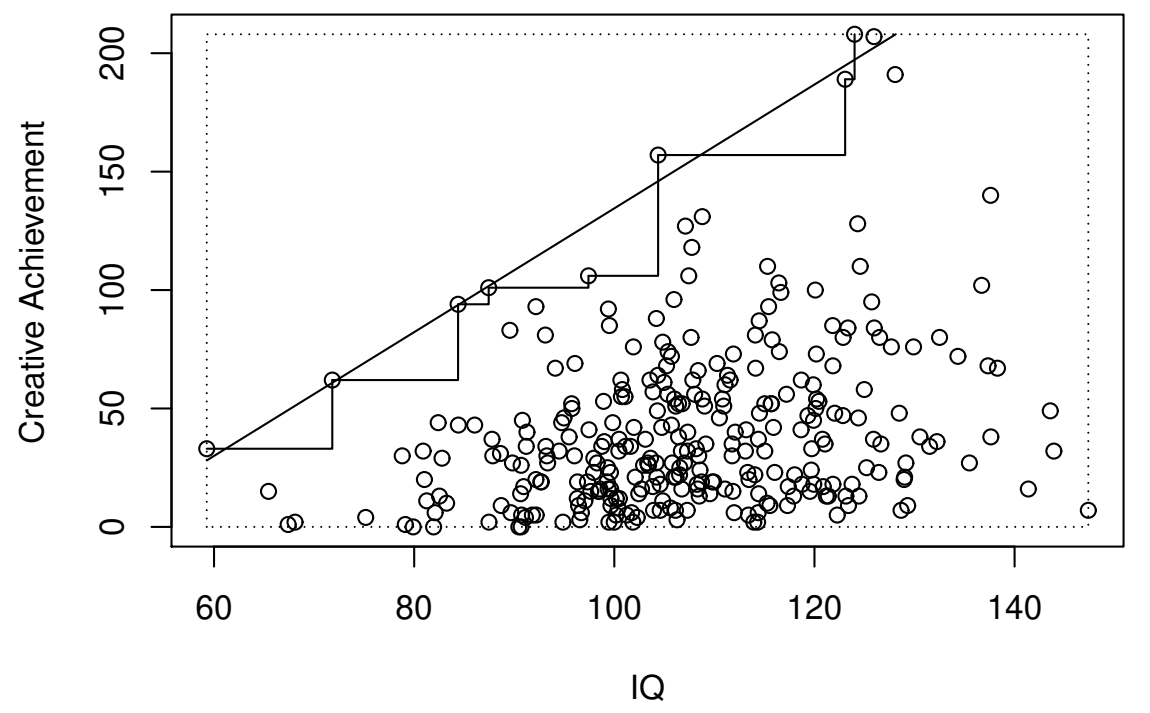

Figure 6. IQ vs. Creative Achievement (CA) from Jauk et al. (2013b), with result from Necessary Condition Analysis (NCA). Stepped lines are the NCA ceiling under CE-FDH; straight line is the NCA ceiling under CR-FDH.

The model fit was visualized via simulation. Creativity values were simulated, using the obtained $\hat{\theta}$ and a resample of IQ, 500 observations, plus random uniform jitter. The simulated creativity values were scaled and shifted to reflect the natural range of the creativity variable. The resulting IQ quantile rank vs. creativity scatterplot overlaid the actual observations with the simulated observations, running $99 \%$ prediction interval, and running mean.

For hypothesis testing, a nonparametric bootstrap resampling, 400 replicates, was used to construct an empirical distribution of $\hat{\theta}$. We break down the minimal hypothesis as

$$
\left\{\begin{array}{l}
a_{1}-a_{0} \geq 0 \\
\left(b_{1}-b_{0}\right)-\left(a_{1}-a_{0}\right)>0,
\end{array}\right.
$$

of which each left-hand side was subjected to a one-tailed test. With a Bonferroni correction to cap the Type I error rate at $5 \%$, the test quantiles were chosen to have quantile rank $2.5 \%$. The alternative hypothesis must be supported for both differences; otherwise, the null hypothesis is retained.

Such procedure is computationally intensive. End to end, the R session took roughly six hours on a computer with $1.9 \mathrm{GHz}$ processor and $2 \mathrm{~GB}$ memory.

\section{Results and discussion}

Table 3 reports the parameter estimates. The visualization of $\hat{\theta}$ is in Figure 7 for IQ vs. CPA and in Figure 8 for IQ vs. CA. The scatterplots validated the model proposed in 


\begin{tabular}{ccccccccc}
\hline \multirow{2}{*}{ Analysis } & \multicolumn{4}{c}{ Estimated values } & & \multicolumn{2}{c}{ Empirical 2.5\% quantiles } \\
\cline { 2 - 4 } \cline { 7 - 8 } & $a_{0}$ & $b_{0}$ & $a_{1}$ & $b_{1}$ & & $a_{1}-a_{0}$ & $\left(b_{1}-b_{0}\right)-\left(a_{1}-a_{0}\right)$ \\
\hline IQ vs. CPA & .142 & .362 & .207 & .389 & & +.038 & -.075 \\
IQ vs. CA & .0005 & .2073 & .0020 & .4553 & & +.0011 & +.1325 \\
\hline
\end{tabular}

Table 3

By creativity value, obtained values of $\hat{\theta}$ and empirical test quantiles for the minimal hypothesis. $C P A=$ Creative Potential Average; $C A=$ Creative Achievement.

that they appear to capture well the structure of the intelligence vs. creativity relationship.

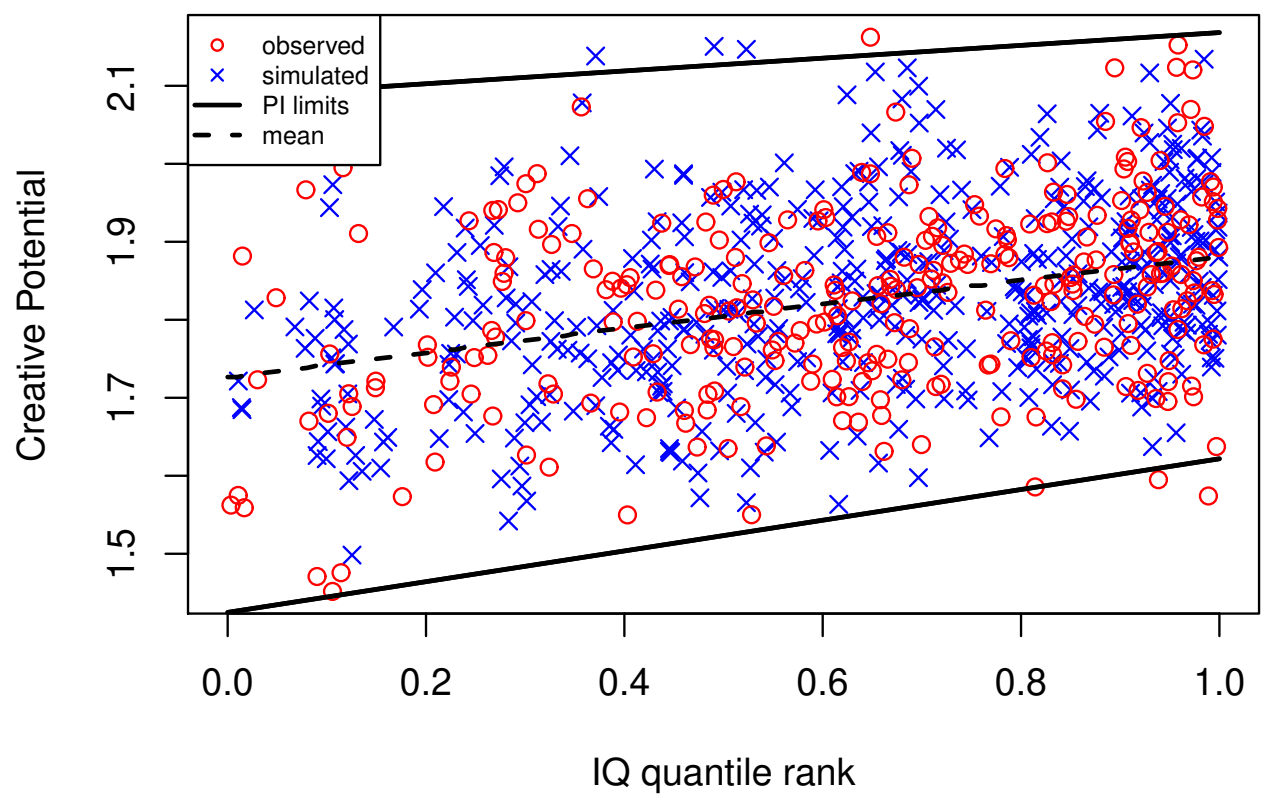

Figure 7. IQ quantile rank vs. Creative Potential Average Originality (CPA) from Jauk et al. (2013b) actual observations, overlaid with $\hat{\theta}=(.142, .362, .207, .389)$ simulated observations, prediction interval (PI) limits, and mean.

Results were mixed. For IQ vs. CA, the theorized relationship was confirmed. In contrast, owing to the nonsignificance of its slope difference $\left(b_{1}-b_{0}\right)-\left(a_{1}-a_{0}\right)$, IQ vs. CPA was still consistent with a conditional distribution merely shifting creativity upwards, without spreading outwards, as IQ increases.

To the best of our knowledge, our analytical treatment satisfies the two desiderata not only in abstract but also with respect to actual data from Jauk et al. (2013b). Our methodology and results demonstrate how carefully specifying a model can accommodate 


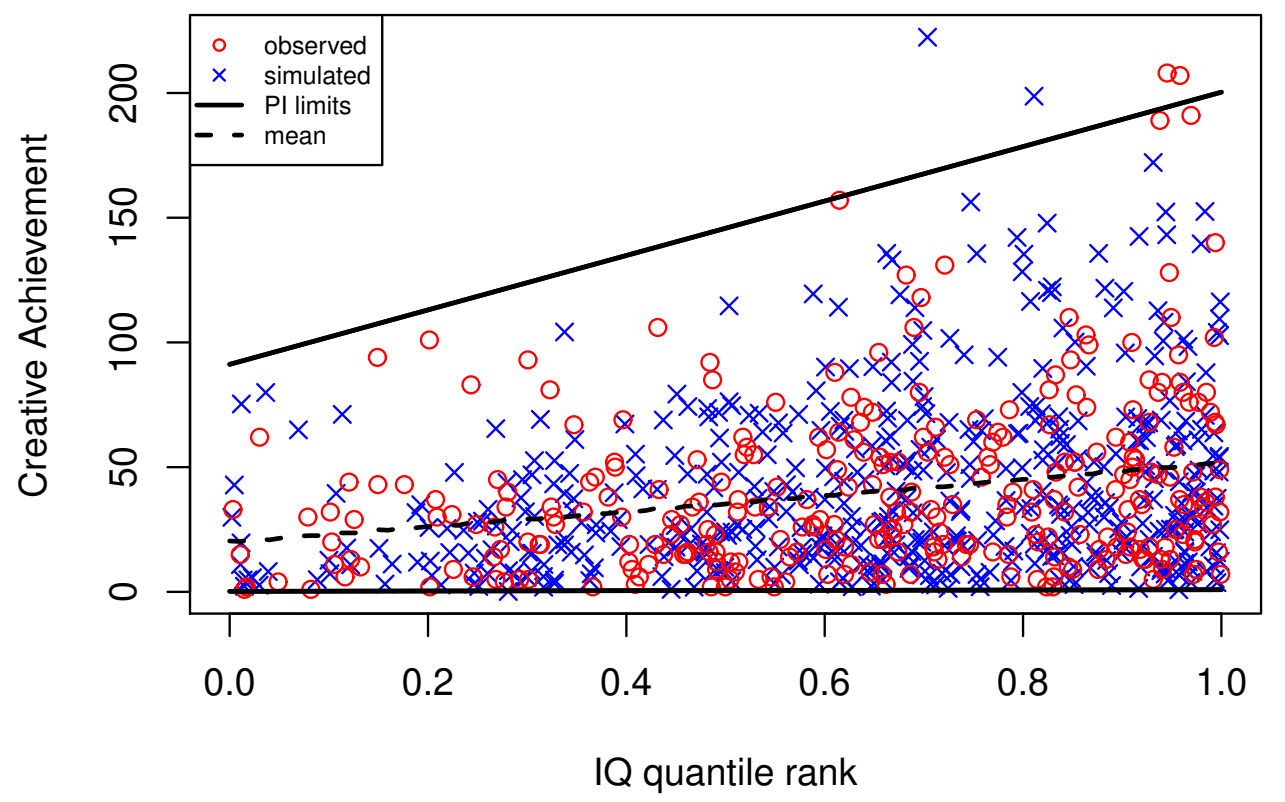

Figure 8. IQ quantile rank vs. Creative Potential Average Originality (CA) from Jauk et al. (2013b) actual observations, overlaid with $\hat{\theta}=(.142, .362, .207, .389)$ simulated observations, prediction interval (PI) limits, and mean.

nuanced assumptions about the subject matter. While regression as conventionally used in the intelligence and creativity literature has not been attentive to the nuances of necessity and sufficiency between variables (Dul, 2016; Karwowski et al., 2016), there is no need to eschew regression altogether. With an appropriate model, regression can tell much about the data-generating process, even the logical role of the predictor as necessary-but-not-sufficient for the response.

On a methodological level, limitations of the present article readily suggest directions for further research. The bivariate distribution of $(X, Y)$ implicit in the model proposed is not as well-understood as tools in more conventional regression methods, compelling us to an analysis with a rather ad hoc character - numerical optimization for estimation, graphical plots for validating model assumptions, and bootstrap resampling for hypothesis testing. Should our approach gain traction, we hope future research to achieve a deeper understanding of this bivariate distribution, towards growing the methodology into a more mature character. But even before analysis of the data, measurement issues must not be ignored. The model proposed is particularly for a double-bounded continuous response, which does not hold for some creativity variables in Karwowski et al. (2016). The model proposed capitalizes on a property of the beta distribution that facilitates matching pairs of quantiles to entire distributions (Van Dorp \& Mazzuchi, 2000) — other distributions may have an analogous property (Cook, 2010), though it is unclear how logical notions can be 
analogously extended to, say, a count variable. Nonetheless, IQ and creativity are only measured, at best, ordinally, and we might speculate that this limitation complicates study of the relationship between intelligence and creativity. For instance, we might speculate that the fact that all of the observations are within a small subrange of CPA's 1-to-4 scale reflects poor quality of measurement that might somehow obscure the underlying relationship.

On a more substantive level, it is incumbent on future research to elaborate on the theory itself. Owing to vagueness, the theorized relationship could not be substantiated per se, compelling us to resort to the minimal hypothesis. Even if the theory were confirmed as in IQ vs. CPA, that fact is itself not very informative, especially as the conditional expectation says very little about the conditional distribution. Keeping the logical motif, a theory to aspire for might have the form

Variables $X_{1}, X_{2}, \ldots, X_{k}$ are individually necessary and jointly sufficient for creativity

for relevant variables $X_{1}, X_{2}, \ldots, X_{k}$-and such a theory would have much more predictive utility, not to mention being effectively a definition of creativity itself. As already found in the literature, besides intelligence, some of these relevant variables may include personality variables (Jauk et al., 2013b; Shi et al., 2017), culture (Mourgues et al., 2016; Shi et al., 2017), and age (Karwowski et al., 2017). As seen in our results, beyond identifying predictors, the operationalization of creativity matters (Jauk et al., 2013b; Karwowski et al., 2016; Karwowski \& Gralewski, 2013; Karwowski et al., 2017) as well. Ultimately, development of an analytical treatment must go hand in hand with development of theory (but see Gigerenzer, 1991), and the goal is to arrive at more specific theories revealing the structure of the phenomenon studied, beyond merely qualitative labels (Cohen, 1994; Marewski \& Olsson, 2009) of variables as "necessary" or "sufficient" for each other.

\section{Conclusion}

The present article is set against a backdrop of a vague theory on the relationship between intelligence and creativity and confused methodology as to how to substantiate this theory. We focused on the methodological problem. Average-based methods, which had been the norm for decades, satisfied only the first desideratum but not the second. NCA, which had been poised to end decades of confusion, under scrutiny satisfied neither desideratum. To satisfy both desiderata, we filled in the gaps left by NCA, specifying a regression model appropriate for a variety of scenarios for which necessity and sufficiency are meaningful. But since the theory is vague, we could test only a minimal hypothesis. Reanalyzing intelligence vs. creativity data (Jauk et al., 2013a, 2013b), the proposed model was found to be appropriate, and the minimal hypothesis was supported for one creativity variable but not the other.

\section{References}

Cade, B. S., \& Noon, B. R. (2003). A gentle introduction to quantile regression for ecologists. Frontiers in Ecology and the Environment, 1(8), 412-420. doi: 10.1890/15409295(2003)001[0412:AGITQR]2.0.CO;2

Casella, G., \& Berger, R. L. (2002). Statistical inference (2nd ed.). Duxbury Press. 
Cohen, J. (1994). The Earth is round ( $\mathrm{p}<.05)$. American Psychologist, 49(12), 997-1003.

Cook, J. D. (2010). Determining distribution parameters from quantiles. (Accessed 2018 February 12 at https://www.johndcook.com/quantiles\%5Fparameters.pdf)

de Vries, J., de Koster, R., Rijsdijk, S., \& Roy, D. (2017). Determinants of safe and productive truck driving: Empirical evidence from long-haul cargo transport. Transportation Research Part E, 97, 113-131. doi: 10.1016/j.tre.2016.11.003

Dul, J. (2016). Necessary condition analysis (NCA): Logic and methodology of "necessary but not sufficient" causality. Organizational Research Methods, 19(1), 10-52. doi: $10.1177 / 1094428115584005$

Fink, M., Haunert, J.-H., Spoerhase, J., \& Wolff, A. (2013). Selecting the aspect ratio of a scatter plot based on its delaunay triangulation. IEEE Transactions on Visualization and Computer Graphics, 19(2), 2326-2335. doi: 10.1109/TVCG.2013.187

Garg, N. (2017). Workplace spirituality and employee well-being: An empirical exploration. Journal of Human Values, 23(2), 1-19. doi: 10.1177/0971685816689741

Gigerenzer, G. (1991). From tools to theories: A heuristic of discovery in cognitive psychology. Psychological Review, 2(2), 254-267. doi: 10.1037/0033-295X.98.2.254

Guilford, J. P. (1967). The nature of human intelligence. New York: McGraw-Hill.

Guilford, J. P., \& Christensen, P. R. (1973). The one-way relationship between creative potential and IQ. Journal of Creative Behavior, 7, 247-252. doi: 10.1002/j.21626057.1973.tb01096.x

Hardegree, G. M. (1999). Symbolic logic: A first course. McGraw-Hill College.

Hasan, P., \& Butcher, H. J. (1966). Creativity and intelligence: A partial replication of with Scottish children of Getzels' and Jackson's study. British Journal of Psychology, 57(1-2), 129-135. doi: 10.1111/j.2044-8295.1966.tb01013.x

Hunt, E. (2011). Human intelligence. Cambridge University Press.

Jauk, E., Benedek, M., Dunst, B., \& Neubauer, A. C. (2013a). Intelligence and doublebounded creativity scores from study on threshold hypothesis via segmented regression [Data set]. Open Science Framework. https://osf.io/hzpgy/.

Jauk, E., Benedek, M., Dunst, B., \& Neubauer, A. C. (2013b). The relationship between intelligence and creativity: New support for the threshold hypothesis by means of empirical breakpoint detection. Intelligence, 41(4), 212-221. doi: 10.1016/j.intell.2013.03.003

Kahneman, D. (2011). Thinking, fast and slow. New York: Farrar, Strauss, and Girroux.

Karwowski, M., Dul, J., Gralewski, J., Jauk, E., Jankowska, D. M., Gajda, A., .. Benedek, M. (2016). Is creativity without intelligence possible? A necessary condition analysis. Intelligence, 57, 105-117. doi: 10.1016/j.intell.2016.04.006

Karwowski, M., \& Gralewski, J. (2013). Threshold hypothesis: Fact or artifact? Thinking Skills and Creativity, 8, 25-33. doi: 10.1016/j.tsc.2012.05.003

Karwowski, M., Kaufman, J. C., Lebuda, I., Szumski, G., \& Firkowska-Mankiewicz, A. (2017). Intelligence in childhood and creative achievements in middle-age: The necessary condition approach. Intelligence, 64, 36-44. doi: 10.1016/j.intell.2017.07.001

Kim, K. H. (2005). Can only intelligent people be creative? The Journal of Secondary Gifted Education, 16 (2-3), 57-66. doi: 10.4219/jsge-2005-473

Liu, Y., \& Wu, Y. (2011). Simultaneous multiple non-crossing quantile regression estimation using kernel constraints. Journal of Nonparametric Statistics, 23(2), 415-437. doi: 


\section{$10.1080 / 10485252.2010 .537336$}

Luther, L., Bonfils, K. A., Firmin, R. L., Buck, K. D., Choi, J., Dimaggio, G., ... Lysaker, P. H. (2017). Metacognition is necessary for the emergence of motivation in people with schizophrenia spectrum disorders: A Necessary Condition Analysis. Journal of Nervous \& Mental Disease, 205(12), 960-966. doi: 10.1097/NMD.0000000000000753

Mackinnon, D. W. (1962). The nature and nurture of creative talent. American Psychologist, 17(7), 484-495. doi: 10.1037/h0046541

Marewski, J. N., \& Olsson, H. (2009). Beyond the null ritual: Formal modeling of psychological processes. Zeitschrift für Psychologie/Journal of Psychology, 217(1), 49-60. doi: 10.1027/0044-3409.217.1.49

Mourgues, C. V., Tan, M., Hein, S., Al-Harbi, K., Aljughaiman, A., \& Grigorenko, E. L. (2016). The relationship between analytical and creative cognitive skills from middle childhood to adolescence: Testing the threshold theory in the Kingdom of Saudi Arabia. Learning and Individual Differences, 52, 137-147. doi: 10.1016/j.lindif.2015.05.005

Muggeo, V. M. R. (2008). segmented: an R package to fit regression models with broken-line relationships. $R$ News, 8(1), 20-25.

Neal, D. J., \& Simons, J. S. (2007). Inference in regression models of heavily skewed alcohol use data: A comparison of ordinary least squares, generalized linear models, and bootstrap resampling. Psychology of Addictive Behaviors, 21(4), 441-452. doi: 0.1037/0893-164X.21.4.441

R Core Team. (2015). R: A language and environment for statistical computing [Computer software manual]. Vienna, Austria. Retrieved from https://www.R-project.org/

Rosenberg, A. S., Knuppe, A. J., \& Braumoeller, B. F. (2017). Unifying the study of asymmetric hypotheses. Political Analysis, 25(3), 381-401. doi: 10.1017/pan.2017.16

Runco, M. A. (2014). Creativity: Theories and themes: Research, development, and practice. Academic Press.

Schubert, D. S. P. (1973). Intelligence as necessary but not sufficient for creativity. The Journal of Genetic Psychology: Research and Theory on Human Development, 122(1), 45-47. doi: 10.1080/00221325.1973.10533169

Shi, B., Wang, L., Wang, J., Zhang, M., \& Xu, L. (2017). Relationship between divergent thinking and intelligence: An empirical study of the Threshold Hypothesis with Chinese children. Frontiers in Psychology, 8, 254. doi: 10.3389/fpsyg.2017.00254

Sligh, A. C., Conners, F. A., \& Roskos-Ewoldsen, B. (2005). Relation of creativity to fluid and crystallized intelligence. Journal of Creative Behavior, 39(2), 123-136. doi: 10.1002/j.2162-6057.2005.tb01254.x

van der Valk, W., Sumo, R., Dul, J., \& Schroeder, R. G. (2016). When are contracts and trust necessary for innovation in buyer-supplier relationships? A Necessary Condition Analysis. Journal of Purchasing and Supply Management, 22(4), 266-277. doi: 10.1016/j.pursup.2016.06.005

Van Dorp, J. R., \& Mazzuchi, T. A. (2000). Solving for the parameters of a beta distribution under two quantile constraints. Journal of Statistical Computation and Simulation, 67, 189-201. doi: 10.1080/00949650008812041

Wilkinson, L., \& the Task Force on Statistical Inference. (1999). Statistical methods in psychology journals: Guidelines and explanations. American Psychologist, 54(8), 
594-604. doi: 10.1037/0003-066X.54.8.594

Yamamoto, K., \& Chimbidis, M. E. (1966). Achievement, intelligence, and creative thinking in fifth grade children: A correlational study. Merrill-Palmer Quarterly of Behavior and Development, 12(3), 233-241. doi: 10.2307/23082661 


\section{Appendix}

Theorem. For fixed $\left(x_{1}, y_{1}\right), \ldots,\left(x_{n}, y_{n}\right)$, let $d_{*}$ be the effect size of a ceiling with perfect accuracy. Then

$$
d_{*} \leq d_{\mathrm{CE}-\mathrm{FDH}}
$$

i.e. CE-FDH maximizes the effect size under the constraint of perfect accuracy.

Proof. Suppose a ceiling $f_{*}$ with perfect accuracy. Then by definition, for all $\left(x_{i}, y_{i}\right)$,

$$
f_{*}\left(x_{i}\right) \geq y_{i}
$$

Let $\mathcal{S}_{1}=\left\{x \in \mathbb{R}: \min _{i} x_{i} \leq x \leq \max _{i} x_{i}\right\}$, and let $g$ be a function, over domain $\mathcal{S}_{1}$, defined as

$$
g(x)=\inf \left\{t \in \mathcal{S}_{1}: f_{\mathrm{CE}-\mathrm{FDH}}(t)=f_{\mathrm{CE}-\mathrm{FDH}}(x)\right\} .
$$

Note that by definition, for $x \in \mathcal{S}_{1}, g(x)$ exists with three properties. First, $f(g(x)) \leq f(x)$ for any ceiling $f$, which is nondecreasing. Second, $f_{\mathrm{CE}-\mathrm{FDH}}(x)=f_{\mathrm{CE}-\mathrm{FDH}}(g(x))$. Third, $g(x)$ is a point where $f_{\mathrm{CE}-\mathrm{FDH}}$ has a jump - then there exists a data point $\left(x_{k}, y_{k}\right)$ such that $x_{k}=g(x)$ and $y_{k}=f_{\mathrm{CE}-\mathrm{FDH}}(g(x))$.

We argue that $f_{*}$ cannot give a more informative bound than $f_{\mathrm{CE}-\mathrm{FDH}}$. Suppose it can, i.e. $f_{*}(c)<f_{\mathrm{CE}-\mathrm{FDH}}(c)$ for some $c \in \mathcal{S}_{1}$. Then applying the first and second abovementioned properties of $g(c)$, it must be that

$$
f_{*}(g(c))<f_{\mathrm{CE}-\mathrm{FDH}}(g(c)),
$$

and, applying the third property, there exists some $\left(x_{k}, y_{k}\right)$ such that

$$
f_{*}\left(x_{k}\right)<y_{k}
$$

which runs contradictory to the definition of perfect accuracy. Thus, it must be the case that

$$
f_{*}(x) \geq f_{\mathrm{CE}-\mathrm{FDH}}(x), \quad x \in \mathcal{S}_{1} .
$$

In other words, $f_{\mathrm{CE}-\mathrm{FDH}}$, under the constraint of perfect accuracy, provides the most informative bound. Then

$$
\begin{aligned}
\frac{\int_{x \in \mathcal{S}_{1}}\left(\max _{i} y_{i}-f_{*}(x)\right) d x}{\mathrm{~A} \mathcal{S}} & \leq \frac{\int_{x \in \mathcal{S}_{1}}\left(\max _{i} y_{i}-f_{\mathrm{CE}-\mathrm{FDH}}(x)\right) d x}{\mathrm{~A} \mathcal{S}} \\
\frac{\mathrm{A}\left\{(x, y) \in \mathcal{S}: y>f_{*}(x)\right\}}{\mathrm{A} \mathcal{S}} & \leq \frac{\mathrm{A}\left\{(x, y) \in \mathcal{S}: y>f_{\mathrm{CE}-\mathrm{FDH}}(x)\right\}}{\mathrm{A} \mathcal{S}} \\
d_{*} & \leq d_{\mathrm{CE}-\mathrm{FDH}} .
\end{aligned}
$$

